\title{
Review: progesterone or progestogens lead to a marginal reduction in premenstrual syndrome symptoms
}

Wyatt K, Dimmock P, Jones P, et al. Efficacy of progesterone and progestogens in management of premenstrual syndrome: systematic review. BMJ 2001 Oct 6;323:776-80.

\section{QUESTION: In women with premenstrual syndrome (PMS), do progesterone or progestogens reduce symptoms?}

\section{Data sources}

Studies were identified by searching Medline, EMBASE/ Excerpta Medica, PsycINFO, the Cochrane Controlled Trials Register, and reference lists, and by contacting pharmaceutical companies.

\section{Study selection}

Studies were included if they were randomised, double blind, placebo controlled studies that included patients with a pretreatment diagnosis of PMS and reported analysable data.

\section{Data extraction}

2 reviewers independently extracted data on participants, dose and preparation of treatment, source of funding, withdrawals, side effects, and outcomes (the main outcome measure was a reduction in overall symptoms of PMS). Disagreements were resolved by discussion with a third reviewer. Reviewers assessed the quality of studies using the Jadad scale and their own 8 point scale.

\section{Main results}

10 studies (531 women) of 14 published trials testing the efficacy of progesterone met the selection criteria. 1 study used both suppositories and oral preparations and was treated as 2 studies in the meta-analysis. The pooled results for 8 studies on suppositories and 3 studies on oral drugs showed a small benefit for progesterone in the reduction of symptoms (table). When results were analysed according to route of administration, a small benefit in favour of placebo over suppositories and in favour of oral micronised progesterone over placebo were seen (table). 3 studies (4 comparisons) of 15 published trials comparing progestogens with placebo met the selection criteria. The progestogens were medroxyprogesterone, $15 \mathrm{mg}$ (1 study); norethisterone, $15 \mathrm{mg}$ (1 study); and dydrogesterone, $20 \mathrm{mg}$ (2 studies). The pooled results showed a marginal benefit for progestogens over placebo in the reduction of symptoms (table). The difference in withdrawals resulting from side effects was not statistically significant for progesterone or progestogens (table).

\section{Conclusion}

In women with premenstrual symptoms, progesterone and progestogens lead to a marginal reduction in symptoms.

\section{COMMENTARY}

The practice of medicine is informed by science and by clinical experience. The many forces that influence patient care can cause deviations from the scientific research that would otherwise support optimal procedures. To illustrate, patients may demand antibiotic treatment for viral infections, compelling clinicians to prescribe medications that do not influence illness course. In other instances, patient care is affected by clinical myth. An example is the view that $\beta$ blockers "cause" depression, an assertion that has no scientific support but could lead to $\beta$ blocker discontinuation in some patients. The utility of progesterone for relief of PMS is another such clinical myth that is variably promoted by physicians and complementary medicine practitioners as well as patients. The logic is facile and thus entrenched: PMS begins when progesterone concentrations drop and thus a relative deficit of progesterone must be causative. Beliefs such as this can be difficult to challenge.

In this study, Wyatt et al selected 10 of the most methodologically sound of 14 articles on progesterone and the 3 best planned and executed of the 15 studies that tested progestogens for moderate to severe PMS. However, even with this pruning, methods varied and the strongest studies are given no greater weight than those with several flaws. This is one of the inherent limitations of meta-analysis: the meta-analytic review can be no better than the studies contributing data. Even considering all studies equally as is done in this review, there was no clinically significant benefit for progesterone or other progestogens. The relative odds of improving after using progesterone or progestogens compared with placebo was only 1.3. This is in contrast to early findings for serotonin transporter blockers that were nearly 7 times more likely to improve symptoms compared with placebo. ${ }^{1}$ These latter statistics are unambiguous and should help guide clinicians' choices even with pressure from colleagues and patients. Prescribing progesterone to a woman with moderate to severe PMS is akin to giving placebo. Certainly, some women will experience a nonspecific response, but the cost is that these patients are not being offered truly effective treatments.

Kimberly Yonkers, MD Yale University New Haven, Connecticut, USA

1 Dimmock PW, Wyatt KM, Jones PW, et al. Efficacy of selective serotonin-reuptake inhibitors in premenstrual syndrome: a systematic review. Lancet 2000;356:1131-6. Review: http:// www.ebmentalhealth.com/cgi/content/full/4/2/43 